\title{
Electrical and optical properties of silicon-doped gallium nitride polycrystalline films
}

\author{
S R BHATTACHARYYA and A K PAL* \\ Department of Instrumentation Science, USIC Building, Jadavpur University, Kolkata 700 032, India
}

MS received 4 July 2007; revised 15 September 2007

\begin{abstract}
Si-doped GaN films in polycrystalline form were deposited on quartz substrates at deposition temperatures ranging from 300-623 $\mathrm{K}$ using r.f. sputtering technique. Electrical, optical and microstructural properties were studied for these films. It was observed that films deposited at room temperature contained mainly hexagonal gallium nitride ( $h$-GaN) while films deposited at $623 \mathrm{~K}$ were predominantly cubic $(c-G a N)$ in nature. The films deposited at intermediate temperatures were found to contain both the hexagonal and cubic phases of GaN. Studies on the variation of conductivity with temperature indicated Mott's hopping for films containing $c$-GaN while Efros and Shklovskii (E-S) hopping within the Coulomb gap was found to dominate the carrier transport mechanism in the films containing $h-G a N$. A crossover from Mott's hopping to E-S hopping in the 'soft' Coulomb gap was noticed with lowering of temperature for films containing mixed phases of GaN. The relative intensity of the PL peak at $\sim 2.73 \mathrm{eV}$ to that for peak at $\sim 3.11 \mathrm{eV}$ appearing due to transitions from deep donor to valence band or shallow acceptors decreased significantly at higher temperature. Variation of band gap showed a bowing behaviour with the amount of cubic phase present in the films.
\end{abstract}

Keywords. Polycrystalline materials; III-V semiconductors; hopping conduction.

\section{Introduction}

The use of polycrystalline semiconductors, in general, had attracted much interest in an expanding variety of applications in electronic and opto-electronic devices (Park et al 2002; Yagi et al 2002; Christie et al 2004). The main technological interest in the polycrystalline based devices lies in its very low production cost and possibility of using low-cost substrates.

A survey of literature indicates that there are not very many studies reported so far on $\mathrm{GaN}$ in polycrystalline form. Only in recent years, some groups explored the feasibility of obtaining polycrystalline GaN layers (Park et al 2002; Yagi et al 2002; Christie et al 2004). It is well known that the polycrystalline films are composed of a large number of crystallites linked by grain boundaries. Potential barriers formed at the grain-grain interfaces can strongly suppress the flow of majority carriers and provide efficient recombination centres for the minority carriers. Thus, the grain boundary scattering would modulate both the electrical mobility and optical transitions. For high resistive films, the measurement of mobility or conductivity becomes difficult, if not impossible. Optical measurements were adopted by some workers to derive information for grain boundary parameters (Red-

*Author for correspondence (msakp2002@yahoo.co.in) field 1963; Dow and Redfield 1970; Bujatti and Marcelja 1972; Maiti et al 1994) in such high resistive films.

In $\mathrm{GaN}$, nitrogen vacancies are known to be energetically most favourable followed by antisites and interstitials. In $p$-type $\mathrm{GaN}$, the nitrogen vacancy (a donor) has the lowest formation energy while in $n$-type GaN the Gavacancy (an acceptor) is preferentially formed. These defects in GaN make effective doping difficult (Morkoc et al 1994; Lester et al 1995). In recent years, improved depositing techniques have allowed some leading groups to grow high quality epilayers and subsequent doping (Davis 1993; Nakamura et al 1996; Pankove 1990; Doverspike and Pankove 1998; Nootz et al 2002; Hageman et al 2003). In the fabrication of blue LED using GaN homojunction, the buffer undoped GaN layer has a carrier concentration, $\sim 10^{17} \mathrm{~cm}^{-3}$, which necessitates the $n$-layer to have high carrier concentration to ensure high emission efficiency. In this regard, Si doping seemed to be a viable alternative to most of the valence-four materials to obtain $n$-type GaN. There are a number of reports on Si-doping in hexagonal GaN (Schubert et al 1997; Lee and Choi 1998; Shmidt et al 1999; Li et al 2001; Yu et al 2004). However, there are not very many studies on Si-doping in c-GaN (Li et al 2001). Speculations are there that Sidoping in epitaxial GaN layer would reduce the density of dislocations (Kisielowski et al 1996; Ruvimov et al 1996). Thus, a careful study on the electrical properties of Si-doped GaN would be relevant. 
Table 1. Here the substrate temperature during deposition $\left(T_{\mathrm{s}}\right)$, bandgap $\left(E_{\mathrm{g}}\right)$, average grain size $(D)$ and surface roughness $\left(s_{0}\right)$ of the GaN films deposited are shown.

\begin{tabular}{lcccc}
\hline $\begin{array}{l}\text { Substrate } \\
\text { temperature, } T_{\mathrm{s}}(\mathrm{K})\end{array}$ & $E_{\mathrm{g}}(\mathrm{eV})$ & $\begin{array}{c}\text { Average grain size }(D) \\
\text { obtained from optical } \\
\text { measurement }(\mathrm{nm})\end{array}$ & $\begin{array}{c}\text { Surface roughness } \\
\left(s_{0}\right)(\mathrm{nm})\end{array}$ & $\Delta E \sigma(\mathrm{meV})$ \\
\hline 300 & $3 \cdot 46$ & 107 & $9 \cdot 47$ & 63 \\
423 & $3 \cdot 38$ & 116 & $10 \cdot 27$ & 59 \\
523 & $3 \cdot 32$ & 118 & $10 \cdot 43$ & 43 \\
623 & $3 \cdot 28$ & 148 & $11 \cdot 32$ & 53 \\
\hline
\end{tabular}

In this communication, we present the electrical and optical properties of Si-doped GaN films in polycrystalline form having different crystallite sizes. The applicability of the existing transport mechanism to describe the observed temperature dependence was also examined.

\section{Experimental}

Polycrystalline Si-doped GaN films were deposited at substrate temperature (within $300-623 \mathrm{~K}$ ) by r.f. sputtering $(13.56 \mathrm{MHz})$ technique using a GaN target containing 1 at \% $\mathrm{Si}$. The Si-doped GaN target was made by mixing appropriate amounts of $\mathrm{Si}(99.995 \%)$ and $\mathrm{GaN}$ powders (99.995\%) supplied by Sigma-Aldrich. The mixture was pressed onto an aluminium target holder $(25 \mathrm{~mm}$ dia). The target was sputtered in argon plasma at a system pressure of $\sim 1.0 \mathrm{~Pa}$. The target to substrate distance was $\sim 3.0 \mathrm{~cm}$. The deposition was carried out for $2 \mathrm{~h}$ at $\mathrm{r}$.f. power $\sim 200 \mathrm{~W}$. Before starting the deposition, the target was pre-sputtered for $\sim 10 \mathrm{~min}$ with a shutter covering the substrate (fused silica). The temperature of the substrate could be measured and controlled $\left( \pm 1^{\circ}\right)$ by a copperconstantan thermocouple acting as the probe of an electronic on/off temperature controller. The thermocouple was placed on a dummy substrate located beside the sample substrate and as such the measured temperature would not differ significantly from that of the substrate. Different sets of films with different polycrystallite sizes were deposited by changing the substrate temperatures $\left(T_{\mathrm{s}}\right)$ during deposition keeping the sputtering time of $\mathrm{GaN}$ constant. The deposition rate was $\sim 8 \mathrm{~nm} / \mathrm{min}$.

Scanning electron microscopy (SEM) and X-ray diffraction (XRD) using $\mathrm{CuK}_{\alpha}$ line $(0 \cdot 154 \mathrm{~nm})$ were used to obtain the microstructural information. The d.c. electrical conductivities $(\sigma)$ of the films were measured in the temperature range $200-300 \mathrm{~K}$ by the four-point probe technique using a constant current source (Advantest) and a Pico ammeter (Keithley 6517A). Optical studies were performed by measuring transmittance and absorbance in the wavelength region, $\lambda=200-900 \mathrm{~nm}$, at room temperature using a spectrophotometer (Hitachi-U3410). The spectra were recorded with a resolution of $\lambda \sim 0.07 \mathrm{~nm}$ along with a photometric accuracy of $\pm 0.3 \%$ for transmittance measurements. Predeposited (evaporated) silver contacts on quartz substrates were used for the d.c. conductivity measurement.

\section{Results and discussion}

\subsection{Microstructural study}

The silicon-doped GaN films (thickness, $\sim 1 \mu \mathrm{m}$ ) were deposited by r.f. sputtering of a GaN target containing 1 at $\%$ $\mathrm{Si}$ onto fused silica substrates kept at different substrate temperatures $\left(T_{\mathrm{s}}\right)$, which are indicated in table 1 . It was observed that films with different grain sizes $(D)$ could be obtained by varying the substrate temperatures during sputtering of the GaN target. The scanning electron micrograph (SEM) of the representative GaN films deposited at substrate temperatures $\sim 300-623 \mathrm{~K}$ are shown in figure 1. The SEM images (figures la-d) reveal that the films are compact with well-dispersed polycrystallites constituting the films. It may be noted here that the SEM picture (1d) of the GaN film deposited at $623 \mathrm{~K}$ showed secondary nucleation on the surface of a continuous film. The grain sizes obtained from the SEM pictures varied between $0 \cdot 10-0 \cdot 15 \mu \mathrm{m}$ with increasing substrate temperature during deposition (table 1). Distribution of grain size obtained from optical measurement (discussed later) is also shown alongside the micrographs.

Figures $2 \mathrm{a}-\mathrm{c}$ show the XRD traces of three representative films deposited at different substrate temperatures during deposition. It may be observed that the XRD traces contained the signature of both the hexagonal and cubic phases of GaN. The spectrum (figure $2 \mathrm{a}$ ) for the film deposited at $300 \mathrm{~K}$ is dominated by broad and relatively low intense peaks indicating smaller grain size in the films. It may also be inferred that the film deposited at lower substrate temperature may contain an admixture of amorphous 'grains' along with crystalline grains. Crystallinity in the films improved with the increase in substrate temperature during deposition. The peaks could be identified as arising for reflections from planes (101) at $2 \theta=$ $36.85^{\circ},(102)$ at $2 \theta=48.26^{\circ},(103)$ at $2 \theta=65.94^{\circ}$ and (202) at $2 \theta=78.5^{\circ}$ for $h-\mathrm{GaN}$. With the increase in substrate temperature during deposition, one may observe (figure $2 \mathrm{~b}$ ) that the peak for reflections from (111) planes of $c-\mathrm{GaN}$ at $2 \theta=38.91^{\circ}$ appeared and the hump corres- 

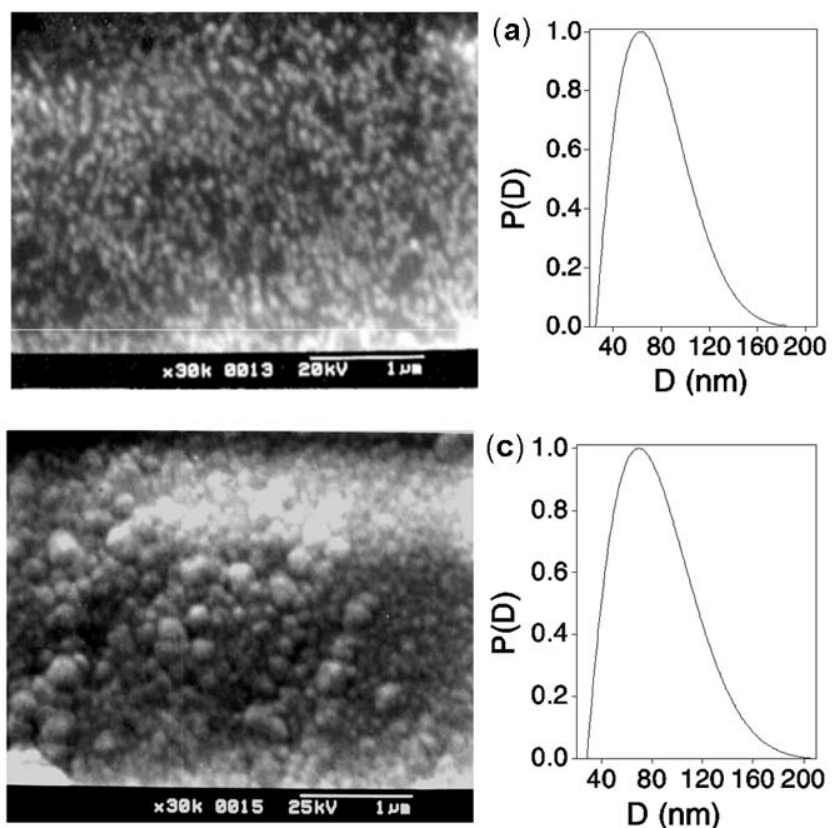
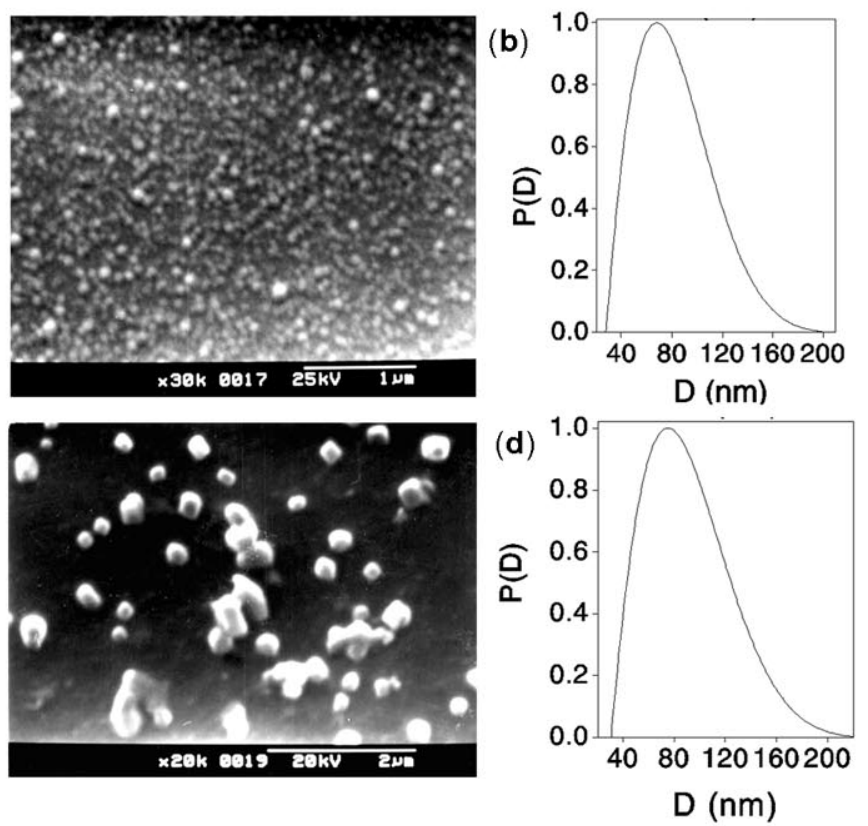

Figure 1. SEM micrographs of four representative Si-doped GaN films deposited at $T_{\mathrm{s}}$ : (a) $300 \mathrm{~K}$, (b) $423 \mathrm{~K}$, (c) $523 \mathrm{~K}$ and (d) $623 \mathrm{~K}$ and the corresponding normalized gaussian grain size distribution plots obtained from optical studies.

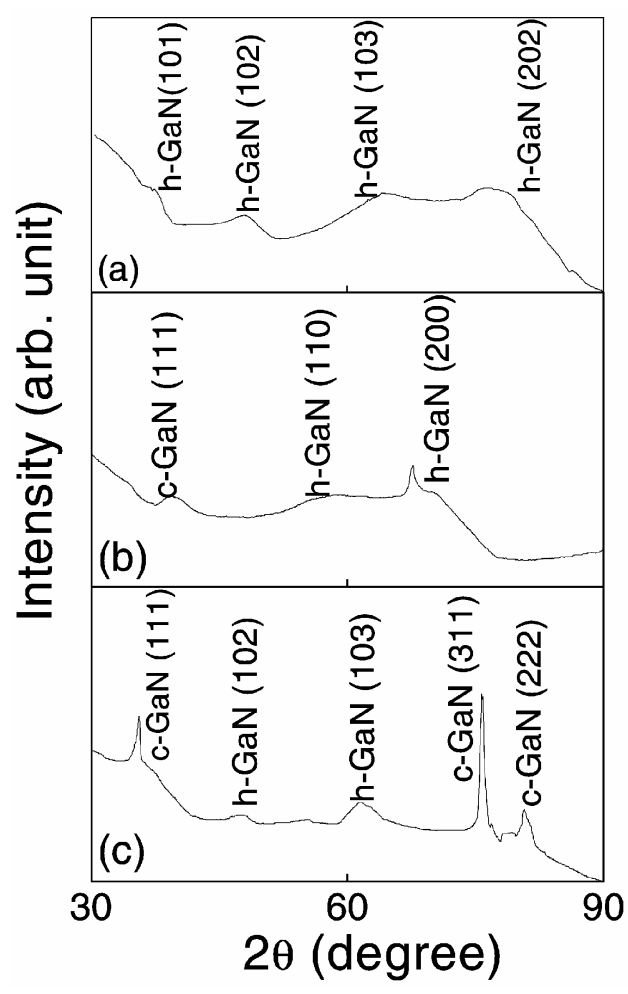

Figure 2. XRD plots for some representative films deposited at (a) $300 \mathrm{~K}$, (b) $523 \mathrm{~K}$ and (c) $623 \mathrm{~K}$

ponding to reflections from (110) and a peak for (200) planes for $h$-GaN could be noticed. The intensity of the peaks gradually increased with the increase in deposition temperature during deposition (figures 2b-c). Similar trends followed as the temperature was increased and film deposited at $623 \mathrm{~K}$ showed (figure $2 \mathrm{c}$ ) strong peaks for reflections from (311) at $2 \theta=79.23^{\circ}$ along with two new peaks of $c-\mathrm{GaN}$ for reflections from (111) and (222) planes at $2 \theta=34.47^{\circ}$ and $2 \theta=83.53^{\circ}$, respectively appeared. One may also observe two small humps corresponding to the reflections from (102) and (103) planes for $h-\mathrm{GaN}$ in the spectra. The relative intensity of the peaks for $c-\mathrm{GaN}$ and $h$-GaN increased significantly and became sharper for films deposited at higher substrate temperature $\left(T_{\mathrm{s}}\right)$ during deposition. As the r.f. power was kept constant, higher substrate temperature during deposition would enhance adatom mobility, which would facilitate the grain growth.

\subsection{Band gap and optical constants}

The absorption coefficients $(\alpha)$ of the Si-doped GaN films were determined by measuring transmittance and reflectance in these films (Manifaciar et al 1977; Bhattacharyya et al 1992). In general, the absorption coefficient $(\alpha)$ may be written as a function of the incident photon energy $(h v)$ so that

$$
\alpha h v=A\left(h v-E_{\mathrm{g}}\right)^{m},
$$

where $m$ would indicate the nature of a particular transition.

$$
\begin{aligned}
& \text { Now, (1) may be rewritten as } \\
& \ln (\alpha h v)=\ln A+m \ln \left(h v-E_{\mathrm{g}}\right)
\end{aligned}
$$


so that

$$
\frac{d[\ln (\alpha h v)]}{d[h v]}=\frac{m}{h v-E_{\mathrm{g}}} .
$$

Equation (3) suggests that a plot of $d[\ln (\alpha h v)] / \mathrm{d}[h v]$ vs $h v$ (figure 3a) will indicate a divergence at $h v=E_{\mathrm{g}}$ from which an approximate value of $E_{\mathrm{g}}$ may be obtained. Once the approximate $E_{\mathrm{g}}$ is found, the value of $m$ can easily be calculated (2) from the slope of the plot of $\ln (\alpha h v)$ vs $\ln \left(h v-E_{\mathrm{g}}\right)$. Figure 3a (inset) shows the plot of $\ln (\alpha h v)$ vs $\ln \left(h v-E_{\mathrm{g}}\right)$ for a representative film, from which one can obtain the value of $m \sim 0.47$ indicating direct transition. The actual band gap $\left(E_{\mathrm{g}}\right)$ for the films was determined by extrapolating the linear portion of the $(\alpha h v)^{2}$ vs $h v$ plot to $(\alpha h v)^{2}=0$. Such a plot for a representative film deposited at $T_{\mathrm{s}}=623 \mathrm{~K}$ showing $E_{\mathrm{g}} \sim 3.28 \mathrm{eV}$ is shown in figure $3 \mathrm{~b}$. The band gaps thus obtained for all the films is given in table 1 and the variation of $E_{\mathrm{g}}$ with the substrate temperatures $\left(T_{\mathrm{s}}\right)$ during deposition is shown in the inset of figure $3 \mathrm{~b}$. It could be observed that the films deposited at a temperature of $\sim 300 \mathrm{~K}$ had a bandgap of $\sim 3.46 \mathrm{eV}$ which is equal to the band gap of $h$-GaN. Films deposited at subsequently higher temperatures indicated gradual lowering of bandgap attaining $\sim 3.2 \mathrm{eV}$ for $c-\mathrm{GaN}$ for films deposited at $T_{\mathrm{s}}>523 \mathrm{~K}$. This clearly collaborates with the observation from the XRD studies that the films deposited at higher temperature $\left(T_{\mathrm{s}}>523 \mathrm{~K}\right)$ contained higher $c-G a N$ phase. At this juncture, it may be worthwhile to indicate that the fall in the absorption curve was sharper in films deposited at higher substrate temperature. This observation is in commensurate with the XRD studies discussed in earlier section.

Since the Si-doped GaN films deposited here consisted of an admixture of hexagonal and cubic phases (Pal Chowdhury et al 2005) and relative percentage of the phases depended on the substrate temperature during deposition, it would be interesting to examine the variation of the above in the films studied here. The variation
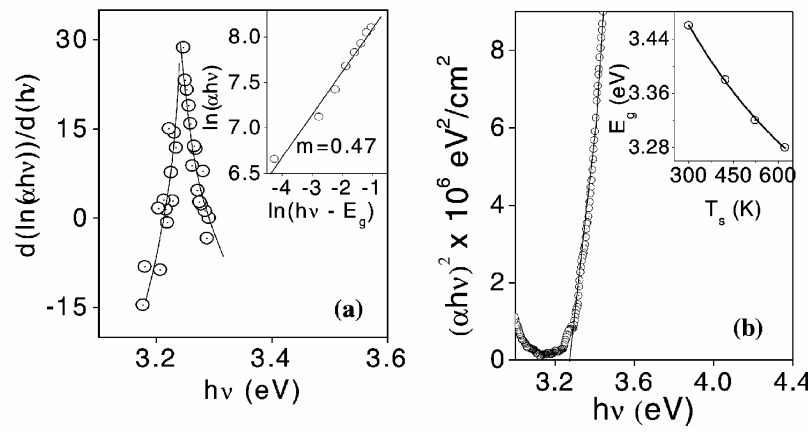

Figure 3. (a) Plot of $d(\ln (\alpha h v)) / d(h v)$ vs $h v$ for a representative film deposited at $623 \mathrm{~K}$. Inset shows $\ln (\alpha h v)$ vs $\ln \left(h v-E_{\mathrm{g}}\right)$ plot and (b) $(\alpha h v)^{2}$ vs $h v$ plot for same film. Inset shows the variation of bandgap $\left(E_{\mathrm{g}}\right)$ for the films deposited at different $T_{\mathrm{s}}$ during deposition. of bandgap could be fruitfully utilized to ascertain the relative amounts of $h$-GaN and $c-\mathrm{GaN}$ present in films deposited at different deposition temperatures during deposition. Now, the variation of bandgap for such films containing two different phases could be expressed by a bowing equation as below

$$
E_{\mathrm{g}}(x)=E_{1}+\left(E_{2}-E_{1}-b\right) x+b x^{2},
$$

where $E_{1}$ and $E_{2}$ are the bandgaps of $h$-GaN and $c$-GaN phases, respectively, $b$ the bowing parameter, $x$ denotes the fractional content of phase having a bandgap, $E_{2}$ i.e. $c$-GaN. As the amount of $h-\mathrm{GaN}$ and $c-\mathrm{GaN}$ are not known, it would be interesting to analyse the variation of bandgap in the light of (4) to derive the value of bowing parameter $(b)$. Apparently it may seem that in order to solve this equation for $b$, we need to know atleast one value of $x$ for which $E_{\mathrm{g}}$ is known in addition to the two trivial solutions at $x=0$ and $x=1$. But, even though the compositions are not known, then also the bowing parameter could be obtained as follows.

Equation (4) is basically a quadratic equation in $x$ and if $E_{1}, E_{2}$ and $E_{\mathrm{g}}(x)$ are known for a given film having $x$ fraction of phase with bandgap, $E_{2}$ and $(1-x)$ fraction of phase with bandgap, $E_{1}$, the solution for $x$ is then given by

$$
\begin{aligned}
x= & {\left[-\left(E_{2}-E_{1}-b\right) \pm \sqrt{ }\left\{\left(E_{2}-E_{1}-b\right)^{2}-4 . b .\right.\right.} \\
& \left.\left.\left(E_{1}-E_{\mathrm{g}}(x)\right)\right\}\right] / 2 b .
\end{aligned}
$$

Since $x$ must always be a real quantity, hence

$$
\left(E_{2}-E_{1}-b\right)^{2}-4 b\left(E_{1}-E_{\mathrm{g}}(x)\right) \geq 0 \text {. }
$$

It is interesting to note that (6) is an equation only in $b$. For the simplest case, approximate values of $b$ can be obtained by setting this equation equal to zero, i.e.

$$
\left(E_{2}-E_{1}-b\right)^{2}-4 b\left(E_{1}-E_{\mathrm{g}}(x)\right)=0 .
$$

The value of bowing parameter thus obtained was $\sim-0.23 \mathrm{eV}$. Once the value of $b$ is obtained as above, one can solve (4), to obtain the value of $x$ corresponding to the different experimental $E_{\mathrm{g}}$ values and the relative amount of different phases of GaN present in the films deposited at different substrate temperatures could be obtained. The calculations showed that the films deposited at $300 \mathrm{~K}$ contained $\sim 100 \% h$-GaN while the films deposited at $623 \mathrm{~K}$ contained $\sim 82 \% c-\mathrm{GaN}$ (figure $4 \mathrm{a}$ ). The values of $x$ (i.e. the percentage of $c-\mathrm{GaN}$ ) computed as above is utilized to indicate the variation of bandgap for films with increasing $c$-GaN content (figure $4 \mathrm{~b}$ ). It may be stressed here that the above computation was based on the assumption that effect of stress in these films would not modulate the optical transitions although stress in these films are quite inherent (Pal Chowdhury et al 2005).

Now, the bandgap $\left(E_{\mathrm{g}}\right)$ of a semiconductor may be related to the effective mass of electron $\left(m^{*}\right)$ in the conduction band (Bhattacharyya et al 1995) as

$$
1 / m^{*}=1+p^{2} / 2 m E_{\mathrm{g}},
$$



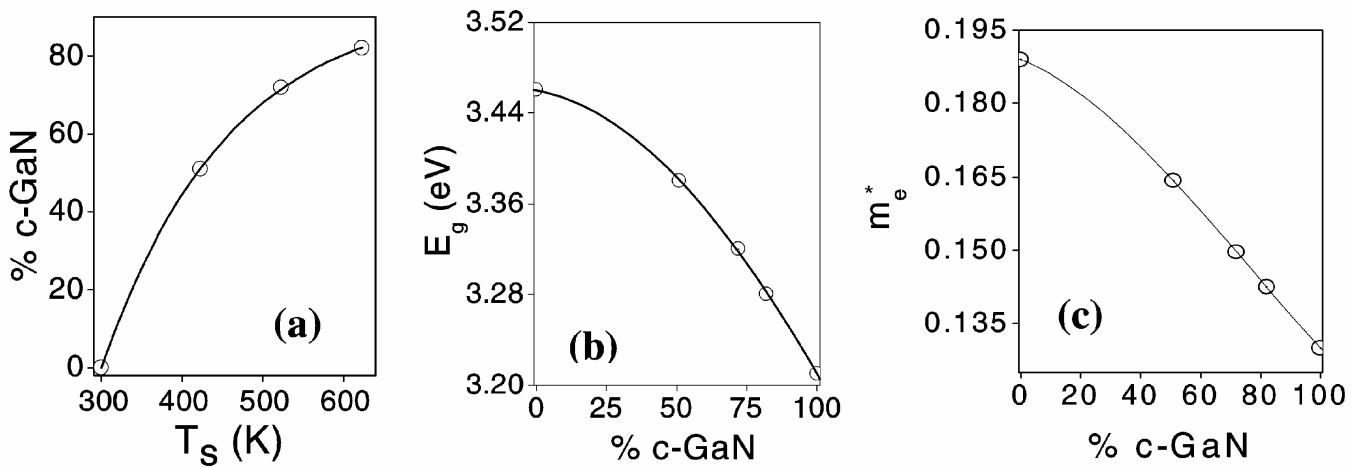

Figure 4. (a) Variation of $\% c$-GaN phase in the Si-doped $\mathrm{GaN}$ films deposited at different $T_{\mathrm{s}}$, (b) variation of bandgap $\left(E_{\mathrm{g}}\right)$ with $c$-GaN content and (c) variation of effective mass of the electron $\left(m_{\mathrm{e}}{ }^{*}\right)$ with the $c-\mathrm{GaN}$ content for films deposited at different $T_{\mathrm{s}}$.

or

$$
E_{\mathrm{g}}=h^{2} f\left(m^{*}\right) / 2 m a^{2},
$$

where $m^{*}=m_{\mathrm{e}} * / m, m$ being the free electron mass, and $p=\hbar G$. As $G$ is the smallest reciprocal lattice vector, $p \sim \hbar / a, a$ being the lattice constant. The value of $f\left(m^{*}\right)$ is given as $m^{*} /\left(1-m^{*}\right)$.

Thus, a bowing equation can be constructed for the effective mass $\left(m^{*}\right)$ of the compound semiconductors having different fractions of the two phases in it, as (Bhattacharyya et al 1995):

$$
m^{*}(x)=m_{1} *+\left(m_{2} *-m_{1} *-b_{1}\right) x+b_{1} x^{2} .
$$

This equation can be treated in a similar manner to obtain the appropriate bowing parameter, $b_{1}$ and hence the effective mass for the films with different fractions of the two phases could also be obtained. The bowing parameters $\left(b_{1}\right)$ for effective mass obtained from (10) was found to be $\sim 2.3 \times 10^{-4}$. A plot depicting the variation of the effective mass $\left(m^{*}\right)$ with the fraction of cubic phase $(x)$ present in the films is shown in figure 4c. The optical constants (refractive index, $n$ and extinction coefficient, $k$ ) of the polycrystalline GaN films were determined from the transmittance and/or reflectance vs wavelength traces by using the standard relations (Manifaciar et al 1977; Bhattacharyya et al 1992). Variation of refractive index and extinction coefficient with $h v$ is shown in figures 5a and $b$, respectively. It may be observed that both the refractive index and the extinction coefficient increased for films deposited at increased substrate temperature during deposition. The refractive index tended to attain the bulk value $(\sim 2 \cdot 3)$ for $c-\mathrm{GaN}$ for films deposited at higher substrate temperature while the absorption coefficient increased significantly for films with higher $c-\mathrm{GaN}$ content. The dielectric constant may be expressed in terms of refractive index, $n$ and extinction coefficient, $k$, as

$$
\varepsilon=\varepsilon_{1}+i \varepsilon_{2},
$$

where the real part of the dielectric constant, $\varepsilon_{1}=n^{2}-k^{2}$ and the imaginary part of the dielectric constant, $\varepsilon_{2}=2 n k$.
We have evaluated the real and imaginary parts of the dielectric constant for all the films studied here from the above relations, which are shown in figures $5 \mathrm{c}$ and $\mathrm{d}$, respectively. It may be observed that the films containing most $c-G a N$ had higher values of the dielectric constants.

\subsection{Grain distribution and surface roughness}

Surface roughness $\left(s_{0}\right)$, which is the rms height fluctuations of the surface irregularities, can be obtained by recording the diffused part of the reflectance $\left(R_{\text {diff }}\right)$ from the film surface (Bhattacharyya et al 1992). We have recorded both the specular $(R)$ and diffused $\left(R_{\text {diff }}\right)$ parts of the reflectance and $s_{0}$ was determined from the variation of $R /\left(R+R_{\text {diff }}\right)$ with wavelength $(\lambda)$ by using the relation (Bhattacharyya et al 1992):

$$
\ln \left[R / R_{0}\right]=-\left(4 \pi s_{0}\right)^{2} / \lambda^{2}+\text { constant }
$$

where $R_{0}=R+R_{\text {diff. }}$ The surface roughness $\left(s_{0}\right)$ for the GaN films deposited at different temperatures was obtained from the slopes of the plots (not shown here) of $\ln \left(R_{0} / R\right)$ vs $1 / \lambda^{2}$. The surface roughness $\left(s_{0}\right)$ varied between 11 and $24 \mathrm{~nm}$ (table 1) and increased for films deposited at higher temperature.

Distribution of grain sizes in these polycrystalline films could also be conveniently evaluated by measuring diffuse $\left(R_{\text {diff }}\right)$ and specular $\left(R_{\mathrm{o}}\right)$ reflections from the film surface (Yu et al 2004) considering a Gaussian distribution of the crystallite size $(D)$

$$
P(D)=\left(D-D_{\min }\right)\left(D_{\max }-D\right) \exp \left(-D^{2} / \delta^{2}\right),
$$

where $D_{\max }$ and $D_{\min }$ are the maximum and minimum diameters of the grains and $\delta$ the half width of the grain distribution, thus, $D_{\max }=\bar{D}+\delta$ and $D_{\min }=\bar{D}-\delta, \bar{D}$ being the average radius. The distribution of grains thus obtained for films deposited at four different temperatures are shown in figure $1(\mathrm{a}-\mathrm{d})$ alongside the corresponding SEM micrographs of the respective films. Variation of 
the average grain diameter of our GaN films (thickness, $\sim 1 \mu \mathrm{m}$ ) deposited at different substrate temperatures, obtained as above, is shown in table 1. This tallied well with that obtained from SEM studies.

\subsection{Photoluminescence studies}

Photoluminescence (PL) measurements were recorded at $78 \mathrm{~K}$ and $300 \mathrm{~K}$ by using a $300 \mathrm{~W}$ xenon arc lamp as the emission source. A Hamamatsu photo multiplier along with a $1 / 4 \mathrm{~m}$ monochromator was used as the detecting system. The spectra were recorded with excitation at $300 \mathrm{~nm}$ radiations for detecting PL peaks in the range 320$550 \mathrm{~nm}$. PL spectra recorded as above at $300 \mathrm{~K}$ and $78 \mathrm{~K}$ for two representative films deposited at $T_{\mathrm{s}} \sim 300 \mathrm{~K}$ and $\sim 623 \mathrm{~K}$ are shown in figures $6 \mathrm{a}, \mathrm{b}$ and $\mathrm{c}, \mathrm{d}$, respectively. The luminescence at $\sim 2.73 \mathrm{eV}$ could be identified as arising due to the transitions between shallow donor arising out of nitrogen vacancy and deep acceptor originating from Ga vacancy with oxygen at a nitrogen site complex $\left(\mathrm{V}_{\mathrm{Ga}}-\mathrm{O}_{\mathrm{N}}\right)$. The origin of the deep donor generally located at $\sim 0.3-0.4 \mathrm{eV}$ below the conduction band may be due to nitrogen vacancy or Ga interstitial related defects. This is the typical signature of near blue luminescence in $\mathrm{GaN}$ having multicomponent nature, which is very sensitive to the exact nature of distribution of the defects (Reshchikov and Morkoc 2005). The peak at $\sim 3.11 \mathrm{eV}$ may be appearing due to transitions from deep donor to valence
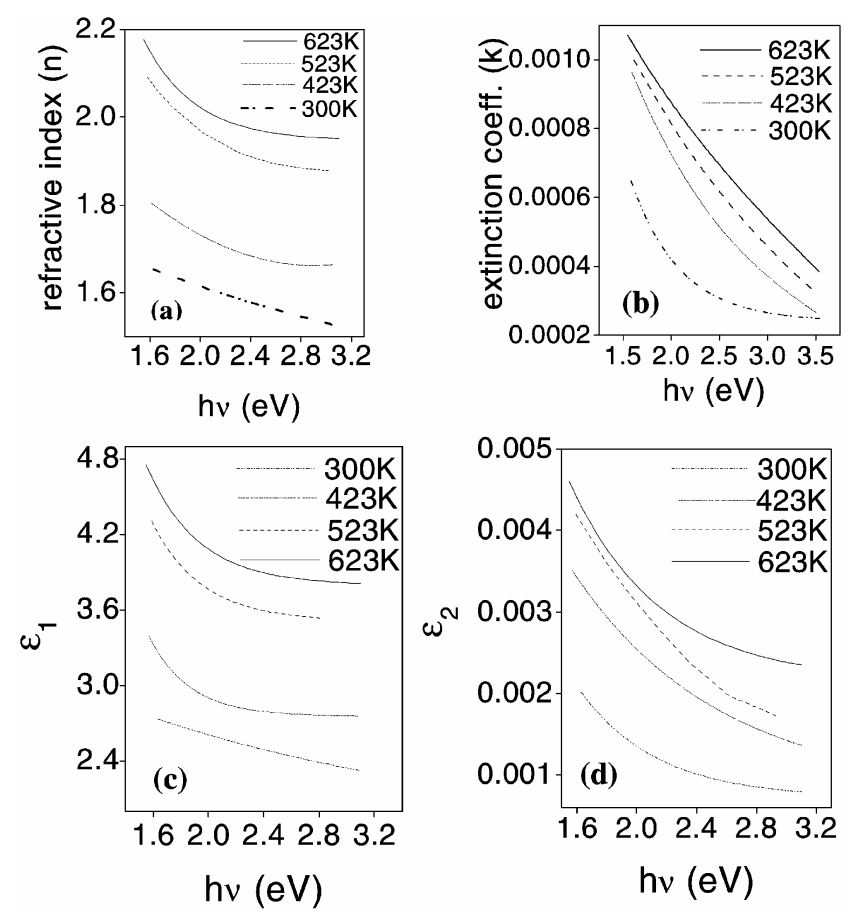

Figure 5. (a) Variation of $n$ with $h v$, (b) variation of $k$ with $h v,(\mathbf{c})$ variation of real and (d) imaginary parts of dielectric constant with $h v$ for four representative Si-doped GaN films. band or shallow acceptors in case of films deposited at $300 \mathrm{~K}$ (figure $4 \mathrm{a}$ ). It may be observed that the relative intensity of peak at $\sim 2.73 \mathrm{eV}$ to that for peak at $\sim 3.11 \mathrm{eV}$ appearing due to transitions from deep donor to valence band or shallow acceptors decreased significantly at higher temperature of measurement. The intensity of the peak at $\sim 3.1 \mathrm{eV}$ increased significantly for films deposited at higher substrate temperature during deposition (figures $4 \mathrm{c}-\mathrm{d})$ and the transitions responsible for this would probably be from shallow donor due to $\mathrm{Si}$ doping at $\sim 16 \mathrm{meV}$ below the conduction band to the valence band. A small peak located at $\sim 3.46 \mathrm{eV}$ in the high energy side for film deposited at $300 \mathrm{~K}$ and $3.28 \mathrm{eV}$ for films deposited at $623 \mathrm{~K}$ for the spectra recorded at $78.4 \mathrm{~K}$ (figures $6 \mathrm{~b}$ and $\mathrm{d}$ ) could be identified as due to excitons bound to neutral donors associated with nitrogen vacancies $\left(D^{\circ}, X\right)$ for $h$-GaN and $c-\mathrm{GaN}$, respectively. This is in conformity with the XRD and optical studies, which indicated that the films deposited at $300 \mathrm{~K}$ and $623 \mathrm{~K}$ contained predominantly $h-\mathrm{GaN}$ and $c-\mathrm{GaN}$ phases. The appearance of an intense sharp peak at $2.64 \mathrm{eV}$ for films deposited at $623 \mathrm{~K}$ may arise due to transitions from deep donor to deep acceptor states. It may be noted here that PL peak located at $\sim 2.6 \mathrm{eV}$ was also observed by Bell et al (2001) in MBE grown GaN layers. They affirmed that the origin
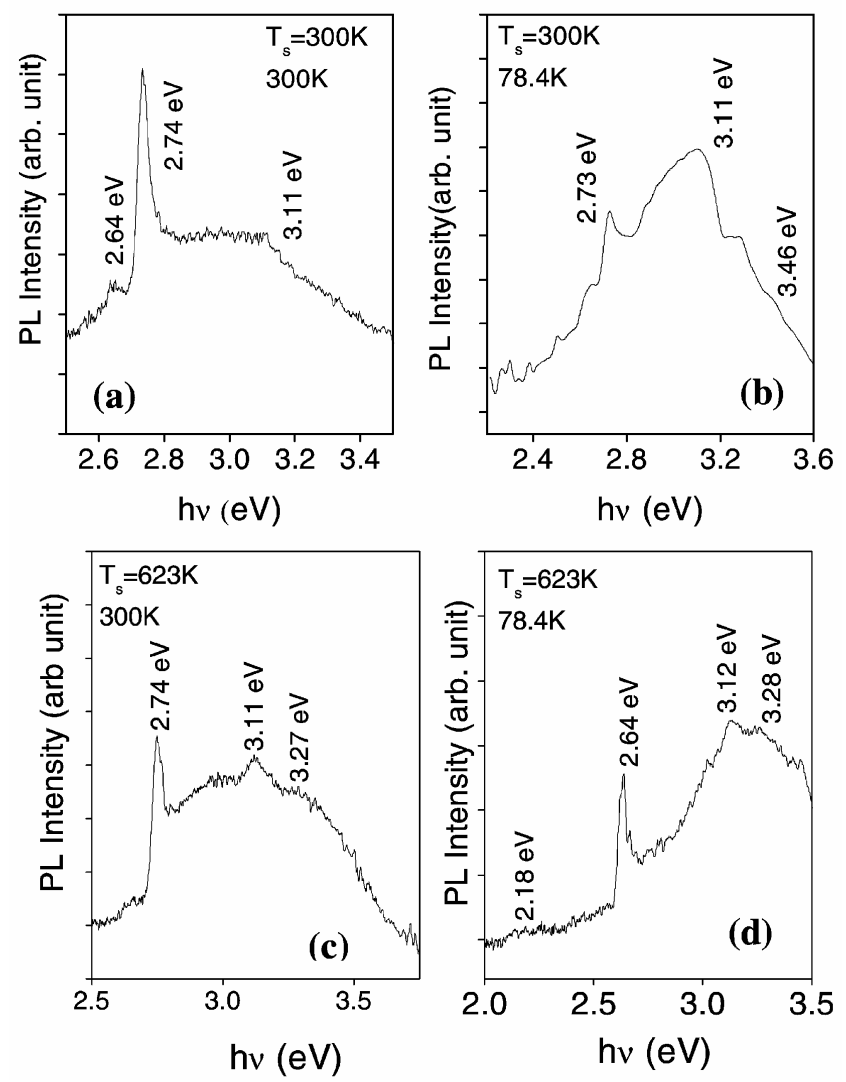

Figure 6. PL spectra for two representative films: (a) and (b) deposited at $T_{\mathrm{s}}=300 \mathrm{~K}$ and recorded at $300 \mathrm{~K}$ and $78.4 \mathrm{~K}$, respectively, (c) and (d) shows the PL spectra for a film deposited at $T_{\mathrm{s}}=623 \mathrm{~K}$ and recorded at $300 \mathrm{~K}$ and $78.4 \mathrm{~K}$, respectively. 
of the deep acceptor at $\sim 0.3 \mathrm{eV}$ was not oxidation related state but was due to annealing induced defect states. Since, in the present case, the film was deposited at higher temperature $(\sim 623 \mathrm{~K})$, induction of such defects would be more probable than that in annealed films.

\subsection{Electrical conductivity measurements}

Electrical conductivity of GaN films deposited as above was measured as a function of temperature ranging from 200-300 K using the standard four-probe technique. The films were highly resistive. Films containing higher percentage of $h-\mathrm{GaN}$ were more resistive than those containing higher $c-\mathrm{GaN}$. It may be noted here that Arrhenius type variation of conductivity with temperature was observed for all the films (figure 7) and the activation energy $\left(\Delta E_{\sigma}\right)$ values obtained from $\ln \sigma$ vs $1 / T$ plots were found (table 1$)$ to be rather small $(\sim 45-63 \mathrm{meV})$. In such cases, the experimentally observed variation of electrical conductivity $(\sigma)$ with temperature is generally analysed in the light of the hopping conduction theories of Mott (Mott and Davis 1971; Mott 1993) and/or Efros-Shklovskii (ES) (Efros and Shklovskii 1975, 1984). The temperature dependence of conductivity is generally described by the expression

$$
\sigma=\sigma_{0}\left[-\left(T_{0} / T\right)^{p}\right]
$$

where the pre-exponential factor, $\sigma_{0}$ may be either independent of $T$ or a slowly varying function of $T$ while $T_{0}\left(=e^{2} / e_{0} e_{\mathrm{r}} a, a^{-1}, e_{0}\right.$ and $e_{\mathrm{r}}$ being the electron localization
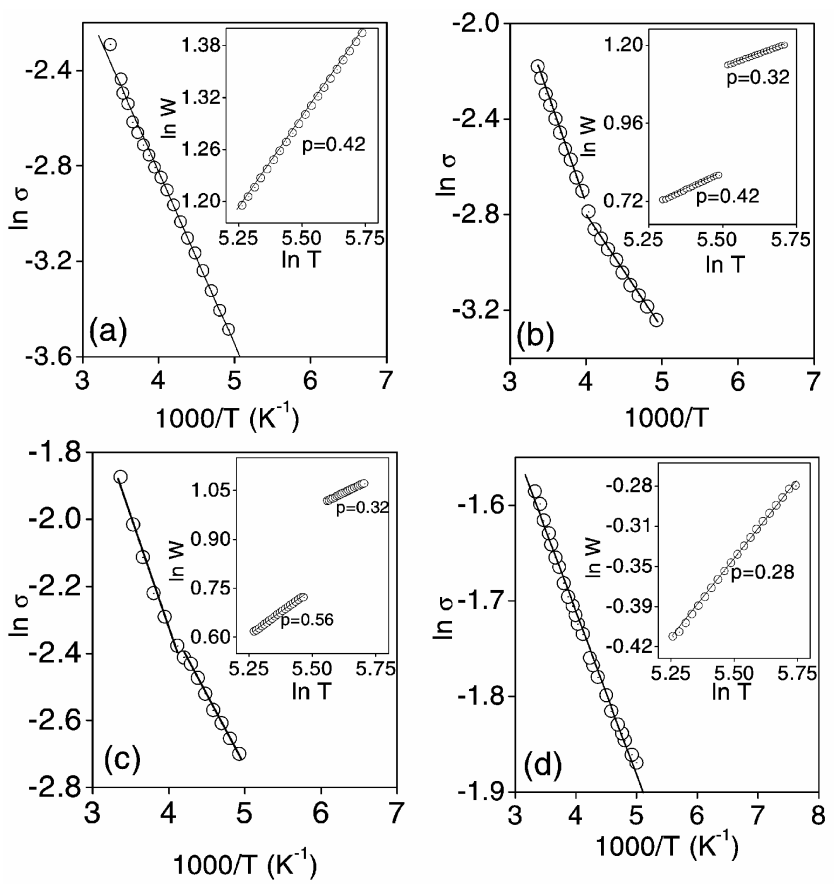

Figure 7. Variation of $\sigma$ with inverse temperature for four representative films deposited at (a) $300 \mathrm{~K}$, (b) $423 \mathrm{~K}$, (c) $523 \mathrm{~K}$ and (d) $623 \mathrm{~K}$. Insets show the corresponding plots of $\ln W(T)$ vs $\ln T$. range, free space permittivity and the dielectric constant of the material, respectively) is a constant of the material. The value of the exponent, $p$, depends critically on the nature of the hopping in the density of states in the bandgap controlling the electron transport processes of the material concerned. In the Mott's variable range hopping regime, the exponent, $p \sim 0 \cdot 25$, while for hopping within the Coulomb gap (ES model), $p$ assumes the value of $\sim 0.5$ either in the homogeneous system of localized interacting electrons or in the form of charging energy in a granular metal-like system (Adkins 1989; Graham et al 1992). It is customary to ascertain the value of $p$ before forcing a fit to the observed variation of the conductivity. Generally, the value of the exponent, $p$, is obtained (Zabrodski and $\mathrm{Zi}$ noveva 1984) by plotting $\ln [W(T)]$ vs $\ln (T)$, where

$$
W(T)=d[\ln \sigma(T)] / d[\ln (T)] .
$$

Equation (14) indicates that the plot should be a straight line and the slope would yield the value of $p$ with high accuracy. It was observed that the value of $p$ ranged between 0.28 and 0.32 and 0.42 and 0.56 in the higher and lower temperature range of measurements for films containing mixed phases of GaN (figures $7 \mathrm{~b}, \mathrm{c}$ ). This would mean that there exists a crossover temperature demarcating the nature of hopping between Mott and ES for such
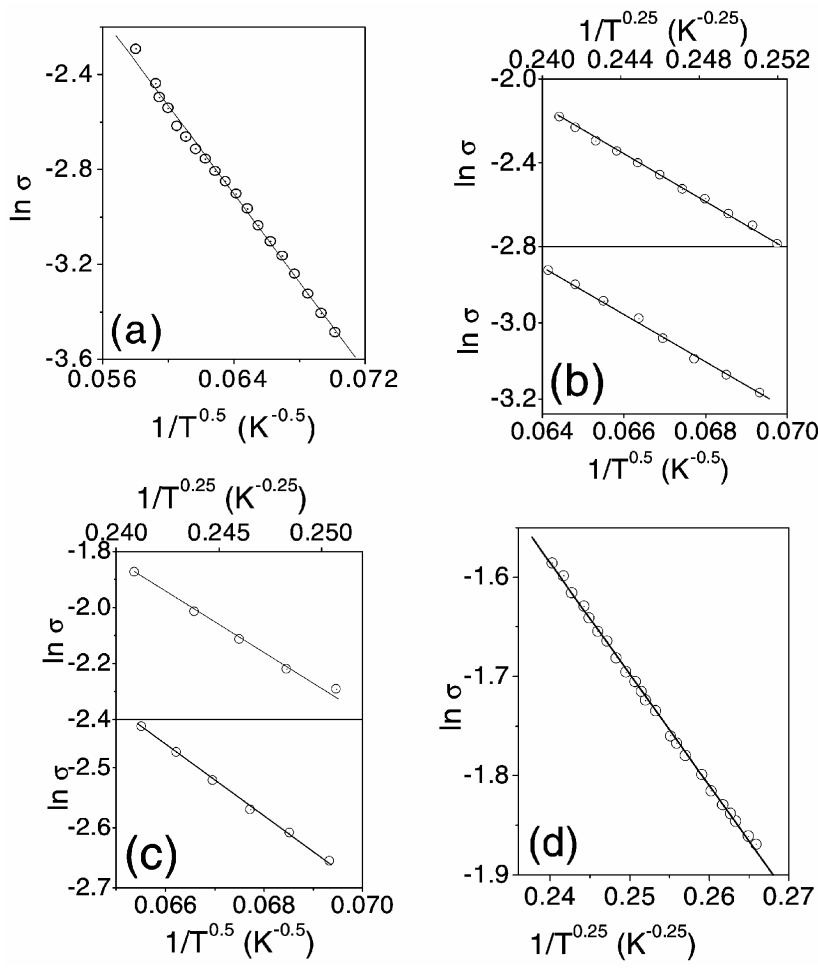

Figure 8. (a) Plot of $\ln \sigma$ vs $1 / T^{0.5}$ for film deposited at $300 \mathrm{~K}$, (b) plot of $\ln \sigma$ vs $1 / T^{0.25}$ and of $\ln \sigma$ vs $1 / T^{0.5}$ for film deposited at $423 \mathrm{~K}$, (c) plot of $\ln \sigma$ vs $1 / T^{0.25}$ and of $\ln \sigma$ vs $1 / T^{0.5}$ for film deposited at $523 \mathrm{~K}$ and (d) plot of $\ln \sigma$ vs $1 / T^{0.25}$ for film deposited at $623 \mathrm{~K}$. 
films. It may be interesting to note that the crossover temperature from ES to Mott shifted to lower temperature with the $c-\mathrm{GaN}$ content in the films so that the films deposited at $623 \mathrm{~K}$ containing most $c-\mathrm{GaN}$ phase would reflect predominant Mott's hopping (figure 7d), while the films deposited at $300 \mathrm{~K}$ with $\sim 100 \% h-\mathrm{GaN}$ would indicate ES hopping within the Coulomb gap (figure 7a). Plots of $\ln [W(T)]$ vs $\ln (T)$ for four representative $\mathrm{GaN}$ films deposited at $300 \mathrm{~K}, 423 \mathrm{~K}, 523 \mathrm{~K}$ and $623 \mathrm{~K}$ are shown in the insets of figures $7 \mathrm{a}-\mathrm{d}$, respectively. It may be observed that films deposited at $300 \mathrm{~K}$ showed $p \sim 0.42$ and those deposited at $623 \mathrm{~K}$ showed $p \sim 0.28$. The film deposited at an intermediate temperature, $\sim 423 \mathrm{~K}$, showed two distinct zones for $p \sim 0.32$ and $p \sim 0.42$ indicating Mott's hopping at higher temperature region and ES hopping for lower temperature region controlling the electron transport process in the film. Thus, one would expect that the temperature variation of electrical conductivity of the film deposited at $300 \mathrm{~K}$ with $\sim 100 \% h$-GaN should be faithfully described by ES model while that for films deposited at $623 \mathrm{~K}$ should be described faithfully by Mott's hopping model. It may be observed that (13) yielded a faithful fit of the experimental data with $p \sim 0.5$ and $p \sim 0.25$ for films deposited at $300 \mathrm{~K}$ and $623 \mathrm{~K}$, respectively as is evident from the plot of $\ln \sigma \mathrm{vs}(1 / T)^{0.5}$ (figure 8a) and $\ln \sigma$ vs $(1 / T)^{0.25}$ (figure 8d). Interestingly, the experimental data for film deposited at intermediate temperatures of $\sim 423 \mathrm{~K}$ and $523 \mathrm{~K}$, fitted well (figures $8 \mathrm{~b}-\mathrm{c}$ ) with $p \sim 0.25$ and 0.5 in the higher temperature and lower temperature domains, respectively indicating the applicability of Mott's and ES relations in these films.

As the electron transport process in films containing predominant $h$-GaN phase is governed by ES model where the hopping in the Coulomb gap region would modulate the conduction process, one may expect the Fermi level may be pinned in the Coulomb gap region and the density of states would then be zero around the Fermi level region. Thus, modulation of conductivity by addition of dopant (here Si) would be difficult for hexagonal GaN than that in films deposited at higher temperature containing predominant $c-\mathrm{GaN}$ phase, where the Fermi level may not be pinned as the electron transport is governed by Mott's hopping. This has been reflected in the magnitude of the electrical conductivity of the films thus deposited. The conductivity of room temperature $(300 \mathrm{~K})$ deposited films was three times lower in magnitude than that for film deposited at $623 \mathrm{~K}$.

3.5a Application of Efros-Shklovskii model for films with predominant $h-G a N$ phase: As mentioned before, in this variable range hopping process, the electrons jump from one localized state to the other in which there is an overlap of the wave functions (Mott and Davis 1971; Mott 1993). The difference in the eigen energies of the two localized states involved in the transition is compensated by the absorption or emission of phonons. As a consequence of the long range Coulomb interaction between the localized state electrons, the density of states (DOS) would tend to zero near the Fermi energy $\left(E_{\mathrm{f}}\right)$ resulting in a soft energy gap corresponding to the parabolic DOS. The idea of the Coulomb gap was first introduced by Efros and Shklovskii $(1975,1984)$ and the corresponding conductivity could be expressed as

$$
\sigma=\sigma_{0} \exp \left[-\left(T_{0} / T\right)^{1 / 2}\right]
$$

where $T_{0}=e^{2} / \varepsilon_{\mathrm{T}} a, a^{-1}$ and $\varepsilon_{\mathrm{r}}$ being the electron localization range and the dielectric constant of the material, respectively.

Figure 8a shows the $\ln \left(\sigma / \sigma_{0}\right)$ vs $1 / T^{0.5}$ plots for a representative Si-doped GaN films deposited at $300 \mathrm{~K}$ for the whole temperature range. Considering the overall uncertainties in measurements (1\%), it may be seen that a satisfactory fit could be obtained for the experimental observation in the whole temperature range. $T_{0}$ values (shown in table 2) derived from the slopes of the above plots were utilized for evaluating several other parameters e.g. hopping energy, hopping distance etc. The temperature dependent optimum hopping distance $\left(R_{\text {hop }}\right)$ is given by

$$
R_{\text {hop }}=0.25 \alpha_{\mathrm{m}}^{-1}\left(T_{0} / T\right)^{1 / 2} \text {. }
$$

Here $\alpha_{\mathrm{m}}$ is the tunneling exponent, which may be obtained from

$$
\alpha_{\mathrm{m}}=k T_{0}\left[\left(\pi g_{2}\right)^{1 / 3}\right] / 10 \cdot 5,
$$

and the value of $g_{2}$ in (4) is given by

$$
g_{2}=3^{8} \pi^{2} \varepsilon_{\mathrm{r}}^{3} \varepsilon_{\mathrm{o}}^{3} / 2^{5} e^{6} .
$$

Here, $\varepsilon_{\mathrm{r}}$ is the dielectric constant of the material and $\varepsilon_{\mathrm{o}}$ the free space permittivity and $e$ the electronic charge. For bulk GaN, we have taken $\varepsilon_{\mathrm{r}}=8 \cdot 11$. For a transport corresponding to 'soft' Coulomb gap $(p=0.5)$, the density of states would have parabolic behaviour in the Coulomb gap, which may be expressed as (Adkins 1989; Graham et al 1992)

$$
g(E)=g_{2}\left(E-E_{\mathrm{f}}\right)^{2} .
$$

It is assumed that the density of states would level off when $g(E)$ equals $g_{0}$ (the Mott density of localized states at the Fermi level which remains unperturbed by the Coulomb interaction), thus defining the Coulomb gap $(\Delta)$ as

$$
g(\Delta / 2)=g_{0},
$$

where $g_{0}$, may be expressed as

$$
g_{0}=\frac{3^{8} \pi^{2} k^{2} \varepsilon_{\mathrm{r}}^{3} \varepsilon_{0}^{3}}{2^{9} e^{6}}\left(T_{0} T^{*}\right),
$$

$T^{*}$ is the temperature at which (17) begins to be satisfied. By using the values of $T_{0}$, we can also compute the $g_{0}$ values (table 2) which could be utilized for evaluating the Coulomb gap $(\Delta)$. 
Table 2. Hopping parameters for Si-doped GaN films for E-S hopping regime.

\begin{tabular}{lccccccc}
\hline$T_{\mathrm{s}}(\mathrm{K})$ & $T_{0} \times 10^{-3}$ & $g_{0} \times 10^{45}\left(\mathrm{~J}^{-1} \mathrm{~m}^{-3}\right)$ & $\alpha_{\mathrm{m}} \times 10^{8}\left(\mathrm{~m}^{-1}\right)$ & $R_{\mathrm{hop}}(\mathrm{nm})$ & $W_{\text {hop }}(\mathrm{meV})$ & $k T(\mathrm{meV})$ & $\Delta(\mathrm{meV})$ \\
\hline 300 & $10 \cdot 1$ & 1.49 & 3.55 & 4 & 75 & 26 & 88 \\
423 & 4.04 & 0.45 & 1.23 & 6 & 56 & 36 & 60 \\
523 & 3.28 & 0.42 & 1.00 & 7 & 56 & 45 & 59 \\
\hline
\end{tabular}

Table 3. Hopping parameters for Si-doped $\mathrm{GaN}$ films for Mott's hopping regime.

\begin{tabular}{lcccc}
\hline$T_{\mathrm{s}}(\mathrm{K})$ & $T_{\mathrm{M}} \times 10^{6}$ & $\alpha_{\mathrm{M}} \times 10^{7}\left(\mathrm{~m}^{-1}\right)$ & $R_{\mathrm{M}}(\mathrm{nm})$ & $W_{\mathrm{M}}(\mathrm{meV})$ \\
\hline 423 & 7.54 & 1.89 & 305 & 108 \\
523 & 4.67 & 1.82 & 267 & 112 \\
623 & 2.65 & 1.17 & 345 & 115 \\
\hline
\end{tabular}

The average hopping energy ( $W_{\text {hop }}$ ) may be estimated from

$$
W_{\text {hop }}=0 \cdot 5 k\left(T_{0} T\right)^{1 / 2} \text {. }
$$

When $W_{\text {opt }}$ becomes comparable or greater than the Coulomb gap $(\Delta)$, there will be transition from ES hopping to Mott hopping and the electrons will use the background or flat density of states. The optimum hopping distance $\left(R_{\text {hop }}\right)$, the mean tunneling exponent $\left(\alpha_{\mathrm{m}}\right)$, Coulomb gap $(\Delta)$ and the optimum hopping energy $\left(W_{\text {hop }}\right)$ were estimated from (16), (17), (20) and (22) in this GaN film and are shown in table 2 .

The explicit expressions for important hopping parameters like the hopping distance $\left(R_{\mathrm{hop}}\right)$, the tunnelling exponent $\left(\alpha_{\mathrm{m}}\right)$ and the optimum hopping energy $\left(W_{\text {hop }}\right)$ associated with E-S hopping within the soft gap is given by (Adkins 1989)

$$
\begin{aligned}
& R_{\text {hop }}=0.25 \alpha_{\mathrm{m}}^{-1}\left(T_{0} / T\right)^{1 / 2}, \\
& \alpha_{\mathrm{m}}=k T_{0}\left(\pi g_{2}\right)^{1 / 3} / 10 \cdot 5, \\
& W_{\text {hop }}=0.5 k\left(T_{0} T\right)^{1 / 2} .
\end{aligned}
$$

Using the above relations, the hopping parameters, computed from the experimental measurements for the GaN films deposited at different temperatures, are shown in table 2 . The width of the Coulomb gap $(\Delta)$ could also be obtained by using the value of $g_{0}$ which is the Mott density of localized states at the Fermi level, unperturbed by the Coulomb interaction.

Now, for the ES hopping model to be applicable in these GaN films, the following criteria are to be satisfied (Pal et al 1994; Mandal et al 1999)

(i) $\quad W_{\text {opt }} \geq k T$ or $T_{0} \geq \mathrm{T}$; $\quad$ (ii) $R_{\text {opt }} \geq d$ and

$$
\text { (iii) } \Delta>W_{\mathrm{opt}}, \Delta>>k T \text {. }
$$

It appears from the values of the different parameters evaluated for our films (table 2) that the first and third criteria in (26) are satisfied i.e. the optimum hopping energy ( $W_{\text {opt }}$ ) is always greater than $k T$ and the hopping energy is less than the width of Coulomb gap which would favour hopping in the localized states within the Coulomb gap. In these films, the second criterion, $R_{\mathrm{opt}} \geq d$ is not satisfied.

\subsection{Application of Mott's model for films with} predominant c-GaN phase

Figure 8d shows the plots of $\ln \sigma \mathrm{vs} 1 / T^{0.25}$ for a representative $\mathrm{GaN}$ film deposited at $623 \mathrm{~K}$, which indicated that Mott relation accounts for the experimental data quite well in the entire temperature range of measurement. The slopes of these plots would give $\beta \alpha_{\mathrm{m}}{ }^{3} / \mathrm{kg}_{0}$. Using $\beta=16$ (Efros and Shklovskii 1984) and the value of $g_{0}$ from table 2, we have estimated the decay length, $\alpha_{\mathrm{m}}{ }^{-1}$ (table 3). It should be mentioned here that there is considerable discrepancy as regards the value of the coefficient $\beta$. The different literature values of $\beta$ reported so far are $16,1.5$ and $24 / \pi(=7.6)$ by Mott (Mott and Davis 1971; Mott 1990, 1993), 18.1 by Castner (1990), 21.1 (Skal and Shklovskii 1974) and 27 (Ortuno and Pollak 1983). We have evaluated the Mott hopping parameters using the relations for the optimum hopping distance $\left(R_{\mathrm{M}}\right)$ and hopping energy $\left(W_{\mathrm{M}}\right)$ (Mott and Davis 1971; Mott 1990, 1993; Banerjee et al 1997), which are shown in table 3.

It may be observed from table 3 that the optimum hopping distance, $R_{\mathrm{M}}$, is larger than the crystallite size and the hopping energy, $W_{\mathrm{M}}$, is significantly larger than the width of the Coulomb gap (table 2). This would mean that hopping of the carriers in the localized states outside the Coulomb gap may become predominant and Mott hopping would govern the electron transport processes, as has been observed here. The decay length $\left(\alpha_{\mathrm{m}}^{-1}\right)$ and the defect density $\left(g_{0}\right)$ did not vary significantly with $T_{\mathrm{s}}$ for different thicknesses (or deposition times) of the GaN films.

3.6a Electron transport in films with mixed $h-G a N$ and $c$-GaN phases: It would be interesting to see how closely the Mott $T^{-0.25}$ relation (Ambegaokar et al 1971; Mott and Davis 1971; Ortuno and Pollak 1983; Mott 1993) and E-S model describe the conductivities in the higher and lower temperature range of measurements for films containing mixed phases of GaN. Figures $8 \mathrm{~b}$ and $\mathrm{c}$ show the plots of $\ln \sigma$ vs $T^{-0.5}$ and $\ln \sigma$ vs $T^{-0.25}$, respectively for a representative film containing mixed phases of $\mathrm{GaN}$. It may be observed that the experimental data for lower temperature of measurements follow E-S model with $p=0.5$ while the data for higher temperature of measurements follow Mott's model with $p=0 \cdot 25$ quite satisfactorily.

\section{Conclusions}

Si-doped GaN polycrystalline films with different grain sizes could be obtained by r.f. sputtering by depositing 
the films on fused silica substrates at different substrate temperatures ranging from 300-623 K during deposition. Increase in grain size with the increase in substrate temperature during deposition was observed. Room temperature deposited films contained $h-\mathrm{GaN}$ and with the increase in substrate temperature during deposition the $c-\mathrm{GaN}$ content increased. Films deposited at $623 \mathrm{~K}$ were found to contain predominant $c-\mathrm{GaN}$ phase. Relative amount of the two phases of $\mathrm{GaN}$ in the films were ascertained from the variation of bandgap with temperature during deposition. Electrical, optical and microstructural properties were measured for these films deposited at different substrate temperatures during deposition. Studies on the variation of conductivity with temperature indicated Mott's hopping for films deposited at higher temperature $(623 \mathrm{~K})$ while Efros and Shklovskii (E-S) hopping within the Coulomb gap was found to dominate the carrier transport process for films deposited at lower temperature $(300 \mathrm{~K})$. A crossover from Mott's hopping in higher temperature region to E-S hopping in the 'soft' Coulomb gap was noticed in films containing mixed phases with lowering of temperature.

The luminescence at $\sim 2.72 \mathrm{eV}$ could be identified as arising due to the transitions between deep donor and induced shallow acceptor by Si impurity without $d$ electron. The origin of the deep donor may be due to nitrogen vacancy or $\mathrm{Ga}$ interstitial related defects. It was observed that the relative intensity of peak at $\sim 2.73 \mathrm{eV}$ to that for peak at $\sim 3.11 \mathrm{eV}$ appearing due to transitions from deep donor to valence band or shallow acceptors decreased significantly at higher temperature.

\section{Acknowledgements}

The authors wish to acknowledge with thanks the financial assistance from the Department of Science and Technology, Government of India, for executing this programme. One of the authors (SRB) wishes to acknowledge with thanks the award of a fellowship by the Council of Scientific and Industrial Research, Government of India.

\section{References}

Adkins C J 1989 J. Phys. Condens. Matter 11253

Ambegaokar V, Halperin B I and Langer J S 1971 Phys. Rev. B4 2612

Banerjee S, Pal R, Maiti A B, Chaudhuri S and Pal A K 1997 Nanostruct. Mater. 8301

Bell A, Harrison I, Korakakis D, Larkins E C, Hayes J M, Kuball M, Grandjean N and Massies J 2001 J. Appl. Phys. 89 1070

Bhattacharyya D, Chaudhuri S and Pal A K 1992 Vacuum 4 313,1201

Bhattacharyya D, Chaudhuri S and Pal A K 1995 Vacuum 461

Bujatti M and Marcelja F 1972 Thin Solid Films 11249

Castner T G 1990 Hopping transport in solids (Amsterdam Elsevier/North Holland) p. 1
Christie V A, Liem S I, Reeves R J, Kennedy V J, Markwitz A and Durbin S M 2004 Curr. Appl. Phys. 4225

Davis R F 1993 J. Condensed Matter 1851

Doverspike K and Pankove J J 1998 Semiconductors and semimetals (San Diego: Academic Press)

Dow J P and Redfield D 1970 Phys. Rev. B1 3358

Efros A L and Shklovskii B I 1975 J. Phys. C8 L49

Efros A L and Shklovskii B I 1984 Electronic properties of doped semiconductors (Berlin: Springer)

Graham M R, Bellingham J R and Adkins C J 1992 Philos. Mag. B65 669

Hageman P R, Schermer J J and Larsen P K 2003 Thin Solid Films 4439

Kisielowski C, Kruger J and Ruvimov S 1996 Phys. Rev. B54 17745

Lee I-H and Choi I-H 1998 J. Appl. Phys. 835787

Lester S D, Ponce F A, Craford M G and Steigerwald D 1995 Appl. Phys. Lett. 661249

Li Z Q, Chen H, Liu H F, Wan L, Huang Q and Zhou J M 2001 J. Cryst. Growth 227-228 420

Maity A B, Chaudhuri S and Pal A K 1994 Phys. Status Solidi b183 185

Mandal S K, Chaudhuri S and Pal A K 1999 Thin Solid Films 357102

Manifaciar J C, de Muricia M, Fillard J P and Vicario E 1977 Thin Solid Films 41127

Morkoc H, Strite S, Gao G B, Lin M E, Sverdlov B and Burns M J 1994 Appl. Phys. 761363

Mott N F 1990 Metal-insulator transitions (London: Taylor and Francis) p. 50

Mott N F 1993 Conduction in non-crystalline materials (Oxford: Clarendon) p. 32

Mott N F and Davis E A 1971 Electronic processes in noncrystalline materials (Oxford: Clarendon Press)

Nakamura S, Senoh M, Iwasa N, Nagahama S, Yamada T, Matsushita T, Kiyoku H and Sugimoto Y 1996 Appl. Phys. Lett. 682105

Nootz G, Schulte A and Chernyak L 2002 Appl. Phys. Lett. 80 1355

Ortuno M and Pollak M 1983 J. Non-Cryst. Solids 59/60 53

Pal Chowdhury M, Roy R K and Bhattacharyya S R 2005 Eur. Phys. J. B48 47

Pal R, Bhattacharyya D, Maiti A B, Chaudhuri S and Pal A K 1994 Nanostruct. Mater. 8329

Pankove J J 1990 MRS Symp. Proc. 162515

Park M et al 2002 J. Appl. Phys. Lett. 811797

Redfield D 1963 Phys. Rev. 130916

Reshchikov M A and Morkoc H 2005 J. Appl. Phys. 97061301

Ruvimov S, Liliental-Weber Z and Suski T 1996 Appl. Phys. Lett. 69990

Schubert E F, Goeptert I D and Griesshaber W 1997 Appl. Phys. Lett. 71921

Shmidt N M et al 1999 Mater. Sci. Engg. B59 195

Skal A S and Shklovskii B I 1974 Sov. Phys. Solid State 16 1190

Yagi S, Suzaki S and Iwanaga T 2002 Jpn J. Appl. Phys. 40 L1349

Yu H B, Chen H, Li D, Han Y J, Zheng X H, Huang Q and Zhou J M 2004 J. Cryst. Growth 26394

Zabrodski A G and Zinoveva K N 1984 Sov. Phys. JETP 59425 